\title{
Chernobyl seed project. Advances in the identification of differentially abundant proteins in a radio-contaminated environment
}

\author{
Namik M. Rashydov ${ }^{1}$ and Martin Hajduch ${ }^{2 *}$ \\ ${ }^{1}$ Department of Biophysics and Radiobiology, Institute of Cell Biology and Genetic Engineering, National Academy of \\ Sciences of Ukraine, Kiev, Ukraine, ${ }^{2}$ Department of Developmental and Reproduction Biology, Institute of Plant Genetics and \\ Biotechnology, Slovak Academy of Sciences, Nitra, Slovakia
}

\section{OPEN ACCESS}

Edited by:

Joshua L. Heazlewood, The University of Melbourne, Australia

Reviewed by: Harriet T. Parsons, University of Copenhagen, Denmark Neil James Willey, University of the West of England, UK

*Correspondence:

Martin Hajduch,

Department of Developmental and Reproduction Biology, Institute of Plant Genetics and Biotechnology,

Slovak Academy of Sciences, Akademicka 2, P.O. Box 39A, Nitra,

Slovakia

hajduch@savba.sk

Specialty section: This article was submitted to Plant Proteomics, a section of the journal Frontiers in Plant Science

Received: 19 December 2014 Accepted: 22 June 2015 Published: 06 July 2015

Citation:

Rashydov NM and Hajduch M (2015) Chernobyl seed project. Advances in the identification of differentially abundant proteins in a radio-contaminated environment.

Front. Plant Sci. 6:493.

doi: 10.3389/fpls.2015.00493
Plants have the ability to grow and successfully reproduce in radio-contaminated environments, which has been highlighted by nuclear accidents at Chernobyl (1986) and Fukushima (2011). The main aim of this article is to summarize the advances of the Chernobyl seed project which has the purpose to provide proteomic characterization of plants grown in the Chernobyl area. We present a summary of comparative proteomic studies on soybean and flax seeds harvested from radio-contaminated Chernobyl areas during two successive generations. Using experimental design developed for radiocontaminated areas, altered abundances of glycine betaine, seed storage proteins, and proteins associated with carbon assimilation into fatty acids were detected. Similar studies in Fukushima radio-contaminated areas might complement these data. The results from these Chernobyl experiments can be viewed in a user-friendly format at a dedicated web-based database freely available at www.chernobylproteomics.sav.sk.

Keywords: soybean, flax, ionizing radiation, ecology, experimental design, seed filling, 2-DE, mass spectrometry

\section{Introduction}

Radioactive minerals have accumulated on the Earth's surface since early Achaean times (3500-4000 million year ago) and probably helped precipitate and concentrate organic carbonrich matter (Parnell, 2004). The first scientific recordings indicating that radioactivity affects living matter dates back to late 19th and early 20th century when Marie Skłodowska-Curie mentioned in her thesis that "The action of radium upon the skin can take place across metal screens, but with weakened effect" (Richards, 1915). Similarly, Henri Becquerel observed negative effects of radioactivity on his own body, after he carried a small tube of impure radium in his pocket for a few hours (Baskerville, 1905). Early experiments on the effect of ionizing radiation (IR) on plants were performed during late 19th and early 20th century (Gager, 1908). It was soon realized that radiation is a powerful mutagen (Nadson and Philippov, 1925), can induce variations within species (Goodspeed and Olson, 1928; Olson and Gilbert, 1928), and can control rates of mutations (Babcock and Collins, 1929).

Plants can easily cope with increased levels of IR. This has been demonstrated in the radiocontaminated Chernobyl (Shkvarnikov, 1990) and Fukushima (Mimura et al., 2014) environments, as well as their successful growth in space (Dubinin et al., 1973). Plant radio-resistance is maybe not surprising since radioactive materials occurred on the Earth's surface when plants 
first appeared during the Mid-Ordovician period, about 460470 million years ago (Wellman and Gray, 2000; Karam and Leslie, 2005). It has also been proposed that present day areas with high-levels of background natural radiation and the reduced levels of plant migration may have both contributed to plant radio-resistance (Moller and Mousseau, 2013). To investigate the molecular aspects of this process in plants, various analyses have been undertaken (Moller and Mousseau, 2015). Recent meta-analysis of 45 published studies on DNA mutations in Chernobyl showed that plant growth in radiocontaminated environment is associated with increased levels of mutation (Moller and Mousseau, 2015). It appears that DNA methylation and increased extra chromosomal homologous recombination events also contribute to successful plant growth in radio-contaminated environments (Kovalchuk et al., 2003, 2004).

However, transcript expression and protein abundance are found to poorly correlate (Chen et al., 2002; Griffin et al., 2002; Orntoft et al., 2002; Pascal et al., 2008; Hornshoj et al., 2009; Hajduch et al., 2010), including plants growing in radio-contaminated areas. Therefore, the complementation of expression studies with proteomics can provide new insight into molecular mechanisms of plant growth in radio-contaminated environments. Indeed, proteome alterations induced by IR are the subject of increased research interest, especially in mammalian systems (Azimzadeh et al., 2014; Leszczynski, 2014). In plants, this is also appears to be the case, as differential abundances of proteins associated with defense and stress responses were detected in leaves harvested from rice grown in the soil taken around Chernobyl reactor site (Rakwal et al., 2009). Importantly, it has been demonstrated that proteome changes increase with irradiation dose; observations were based on the analysis of X-rays irradiated plantlets of the reference plant Arabidopsis thaliana (Gicquel et al., 2011).

\section{Experimental Design of the Chernobyl Seed Project}

Experimental design for ecological field experiments should include several experimental fields to avoid pseudoreplication (Hurlbert, 1984). However, it is often difficult to establish and manage several experimental fields in heavily controlled radio-contaminated areas. Therefore, experimental design for Chernobyl (Figure 1) was modified and included (i) two non-radioactive fields (control) and one radio-contaminated experimental field (Supplementary Figures S1A,B), (ii) two plant species, and (iii) two successive years. An important aspect of this experimental design (Figure 1) is the changed location of the non-radioactive field after the first year. The logic behind this is to exclude alterations related to the differences between experimental fields (soil, pests, weather, etc).

In 2007, local varieties of soybean (Glycine max [L.] Merr., variety Soniachna) and flax (Linum usitatissimum, L., variety Kyivskyi) were sown in radio-contaminated experimental fields (soil radioactivity $20650 \pm 1050 \mathrm{~Bq} \cdot \mathrm{kg}^{-1}$ of ${ }^{137} \mathrm{Cs}$, and $5180 \pm 550$ Bq. $\mathrm{kg}^{-1}$ of ${ }^{90} \mathrm{Sr}$ ) located near the village Chistogalovka approximately $5 \mathrm{~km}$ from the Chernobyl Nuclear Power Plant (CNPP). The non-radioactive control experimental field (1350 $\pm 75 \mathrm{~Bq} \cdot \mathrm{kg}^{-1}$ for ${ }^{137} \mathrm{Cs}$ and $490 \pm 60 \mathrm{~Bq} \cdot \mathrm{kg}^{-1}$ for ${ }^{90} \mathrm{Sr}$ ) was established near Zhukin, a village approximately $100 \mathrm{~km}$ from CNPP (Supplementary Figure S1A). Soybean and flax seeds were harvested and mature seed proteomes comparatively analyzed in biological triplicate (Figure 1). In 2008, soybean and flax seeds harvested from the first generation of plants were sown onto the same radio-contaminated field, but a different non-radioactive field in the Chernobyl area (Supplementary Figure S1B), to obtain the second generation of seeds. A new non-radioactive experimental field was established directly in the town of Chernobyl, in an area with soil radioactivity of $1414 \pm 71 \mathrm{~Bq} \cdot \mathrm{kg}^{-1}$ of ${ }^{137} \mathrm{Cs}$ and $550 \pm 55 \mathrm{~Bq} \cdot \mathrm{kg}^{-1}$ of ${ }^{90} \mathrm{Sr}$ (Supplementary Figure S1B). The Chernobyl area is characterized by sod-podzolic soil (pH5.6-pH6.6, 12\% clay, 2.0\% organic compounds) which is a typical soil in the Ukrainian region of Polessia. Generally, in this area, the content of aleurite (silt) and pelitic soil ranges from 20 to $30 \%$ (Rashydov and Malinovskiy, 2002).

\section{Advances in the Establishment of Protein Abundance Profiles and Web-Based Database}

In soybeans of the first generation, only 9.2\% 2-DE spots, out of 698 quantified, were found differentially abundant between mature seeds harvested from non-radioactive and radiocontaminated Chernobyl areas (Danchenko et al., 2009). Similar to this, the analysis of the first generation of mature flax seeds showed differential abundance only in about $4.9 \%$ of resolved features from 720 quantified 2-DE spots (Klubicova et al., 2010). However, the results from these initial soybean and flax generations do not represent a large enough dataset upon which it is possible to base solid conclusions; it appears that growth in radio-contaminated environments has a relatively small effect on the seed proteome. Similar effects of IR have been previously shown on animal proteomes (Park et al., 2006; Guipaud et al., 2007) and support the notion that the exposure to low levels of IR do not significantly alter overall metabolism. Such speculation may be further supported by a study on the roots of the reference plant Arabidopsis thaliana under low levels of ${ }^{137} \mathrm{Cs}$, where only a small percentage of the transcriptome was differentially expressed (Sahr et al., 2005).

In order to provide a more detailed insight into the seed proteome in radio-contaminated environments, protein abundances profiles were established from developing soybean and flax seeds (Figure 2) from the second generation which were harvested from both Chernobyl experimental fields (Supplementary Figure S1B). Protein abundance profiles are capable of comprehensively characterizing protein abundances during seed filling. The approach has been used successfully in soybean (Hajduch et al., 2005), canola (Hajduch et al., 2006), castor (Houston et al., 2009), and Arabidopsis (Hajduch et al., 2010). In these Chernobyl studies, protein abundance 


\section{Soybean}

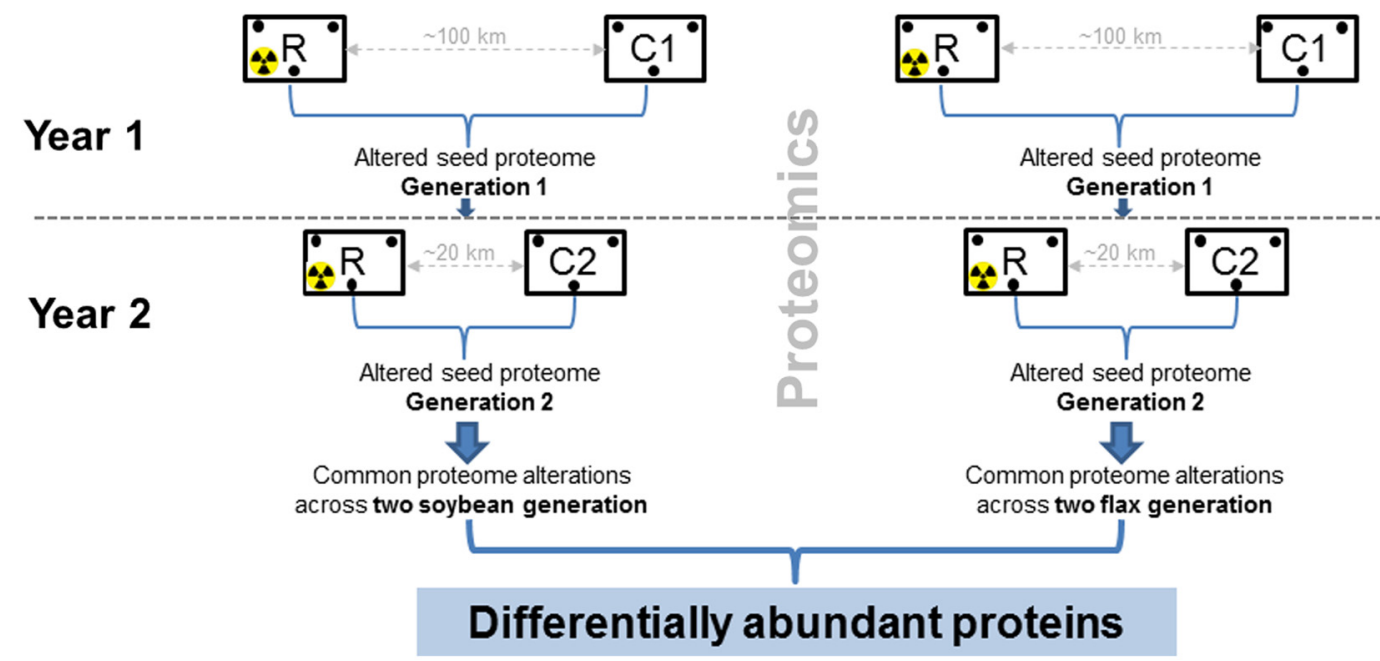

FIGURE 1 | Experimental design in the Chernobyl area during the two-year proteomic survey. In the first year, local varieties of soybean (Glycine max [L.] Merr., variety Soniachna) and flax (Linum usitatissimum, L., variety Kyivskyi) were planted in radio-contaminated $(R)$ and non-radioactive (C1) experimental fields in the Chernobyl area (Supplementary Figure S1). Seeds were harvested in biological triplicate and subjected to proteomic analyses. The following year, seeds not used for the analyses were planted into the same radio-contaminated field (R), but different non-radioactive (C2) experimental fields to obtain seeds from the second generation. To exclude alterations in seed proteomes related to field locations, only those differentially abundant proteins commonly observed across the two soybean and flax generations were considered.

\section{Seed filling in Chernobyl}

Non-radioactive field

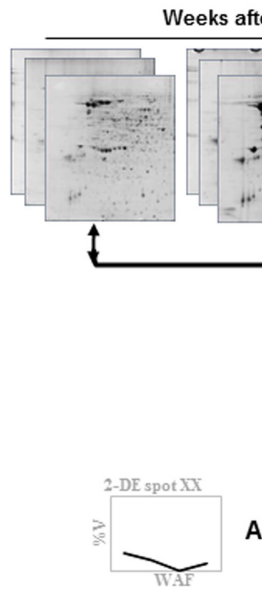

eks after flowering

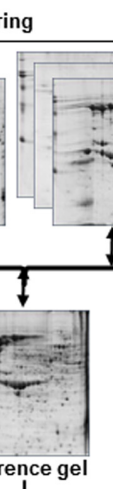

$\downarrow$

Abundance profiles

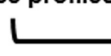

mature

\section{Radio-contaminated field}
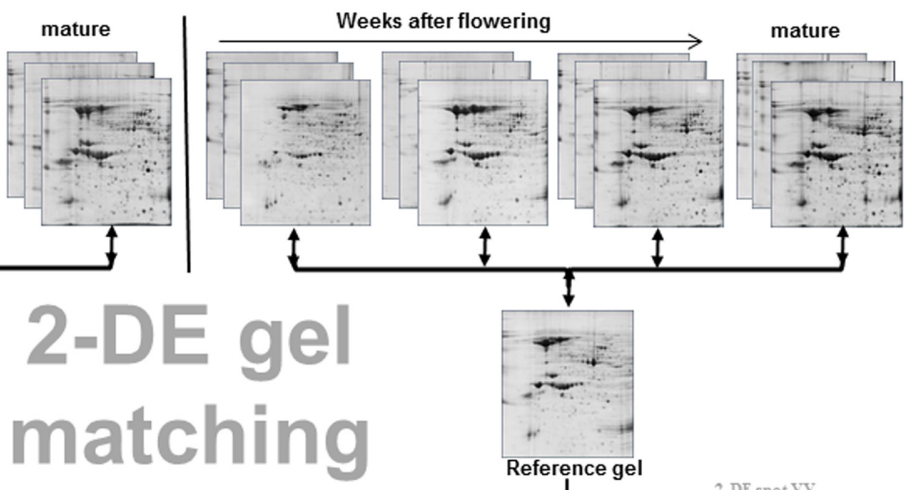

matching

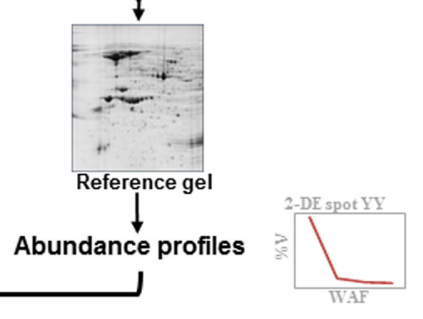

Joint abundance profiles

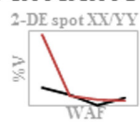

Non-radioactive

Radio-contaminated

FIGURE 2 | Schematic overview on the establishment of protein abundance profiles - modified from Klubicova et al. (2012a). Briefly, developing soybean seeds were harvested at 4, 5, 6 weeks after flowering (WAF) (flax seeds at 2, 4, and 6 WAF) and at a mature stage from plants grown in non-radioactive and radio-contaminated Chernobyl experimental fields (Supplementary Figure S1). Isolated total protein was resolved by two-dimensional protein electrophoresis (2-DE) in biological triplicate. The 2-DE gels were matched to the pooled (reference) gels using ImageMaster 4.9 software. Finally, abundance profiles from both experimental fields were matched and joint abundance profiles, i.e., profiles for the same spot across seed filling in non-radioactive and radio-contaminated experimental fields, were established. 
profiles were first established for each experimental field and then matched to obtain joint abundance profiles (Figure 2). Using this approach, it was possible to provide a detailed overview of protein abundances during seed filling in soybean (Klubicova et al., 2012a) and flax (Klubicova et al., 2010) across both experimental fields. For instance, it was revealed that $\beta$-conclycinin significantly decreased during seed filling in radio-contaminated areas in the second soybean generation (Klubicova et al., 2012a). These analyses also revealed alterations of proteins associated with carbon metabolism in the cytoplasm and plastids and to the carboxylic acid cycle in the mitochondria (Klubicova et al., 2012a). In flax, increased abundance of proteins associated with isocitrate dehydrogenation, L-malate decarboxylation, pyruvate biosynthesis, and ethanol oxidation to acetaldehyde were detected at early stages of seed filling (Klubicova et al., 2013).

The data from these experiments can be viewed in a userfriendly format at dedicated web-based database that is freely available at www.chernobylproteomics.sav.sk. The aim of this online data depository is to allow scientific community (but also general public) to access the data from this project in user-friendly format. At the time of the database establishment (Klubicova et al., 2012b) the database contained the data from first, second soybean and first flax generation. Since then, the data from second flax (Klubicova et al., 2013) and third (Gabrisova et al., in review) generations were uploaded.

\section{Chernobyl Seed Project Suggested the Identity of Proteins Putatively Associated with Plant Growth in Radio-Contaminated Environments}

The aim of these studies was to detect alterations in seed proteomes related to the radio-contaminated environment. However, the alterations in seed proteomes described above might also be associated with the differences between the experimental fields (soil, pests, weather etc). To exclude this possibility, data were further analyzed and alterations common for both plant generations and plant species identified.

Altered abundance of enzymes associated with the glycine betaine biosynthetic pathway was jointly detected in the first generation of soybean (Danchenko et al., 2009) and flax (Klubicova et al., 2010). It is tempting to speculate that glycine betaine is involved at early stages of plant response toward the radio-contaminated environment. Interestingly, the involvement of glycine betaine in protection against IR was shown previously in human blood (Monobe et al., 2005). Since plants with altered levels of glycine betaine have already been produced (Waditee et al., 2007) it should be possible to directly test the putative protective role of glycine betaine in radio-contaminated environments.

The mobilization of seed storage proteins (SSP) and alteration of proteins associated with carbon assimilation and fatty acid metabolism were observed jointly in both generations of soybean and flax. These data support the notion that SSPs are involved in seed defense against various threats, as has been shown previously with their role in defense against Bruchids (Sales et al., 2000). Interestingly, the application of salicylic acid during germination Arabidopsis thaliana resulted in mobilization of SSPs (Rajjou et al., 2006). Furthermore, it has been proposed that class $2 S$ albumin SSPs are defensive proteins (Regente and De La Canal, 2001), while salt stress has been shown to alter the abundance of $\beta$-conglycinin SSP (Aghaei et al., 2009).

An interesting aspect of these Chernobyl studies are differential abundance of proteins associated with carbon assimilation and fatty acid metabolism in both generations of soybean and flax. As a result of this, the second generation of soybean (Klubicova et al., 2012a) and flax (Klubicova et al., 2013) showed altered total oil content in mature seeds. However, additional studies are needed to determine whether altered seed oil content is the result of genetic mutation or has an epigenetic or posttranslational explanation.

\section{Studies in Fukushima Radio-Contaminated Environment}

Similar to the disaster at the CNPP in 1986, the accident at the Fukushima Daiichi Nuclear Power Plant in 2011 contaminated large areas with radioactivity (Buesseler et al., 2011; Kinoshita et al., 2011; Yasunari et al., 2011). Unfortunately, nuclear accidents provide unexpected justifications for research aimed at understanding plant survival and adaptation in radiocontaminated environments. Indeed, Hayashi at al. (2014) performed a pioneering study in the Fukushima radiocontaminated areas through the investigation of rice seedlings under continuous low-dose radiation. This study provided an overview of the transcriptome response in rice toward low level of gamma radiation and identified large numbers of genes with altered expression patterns (Hayashi et al., 2014).

It will be interesting to compare results from the Chernobyl studies using similar experimental setups in the Fukushima radio-contaminated area. The web based database (chernobylproteomics.sav.sk) might be a good tool for quick data comparison. Ideally, follow-up studies in Fukushima should include several non-radioactive and radio-contaminated experimental fields to avoid pseudoreplication (Hurlbert, 1984). If this is not feasible due to restricted/closed areas, an experimental design for radio-contaminated areas presented in this current study (Figure 1) could be applied.

\section{Conclusion}

The outcome of these Chernobyl studies was the identification of several proteins with differentially abundances in soybean and flax seeds harvested during two successive generations. It is tempting to speculate that these proteins are associated with plant growth and adaptation in radio-contaminated environments. However, follow-up studies in both the Chernobyl and Fukushima radio-contaminated areas are required to further develop these hypotheses. 


\section{Acknowledgments}

This research was supported by the Slovak Research and Development Agency (APVV-0740-11), by European Community under Project no 26220220180: Building Research Centre "AgroBioTech," and Seventh Framework Program of the European Union (IRSES-GA-2013-612587). This research was also partially supported by the Scientific Grant Agency of the Ministry of the Slovak Republic and Slovak Academy of Sciences (VEGA-2/0016/14).

\section{References}

Aghaei, K., Ehsanpour, A. A., Shah, A. H., and Komatsu, S. (2009). Proteome analysis of soybean hypocotyl and root under salt stress. Amino Acids 36, 91-98. doi: 10.1007/s00726-008-0036-7

Azimzadeh, O., Atkinson, M. J., and Tapio, S. (2014). Proteomics in radiation research: present status and future perspectives. Radiat. Environ. Biophys. 53, 31-38. doi: 10.1007/s00411-013-0495-4

Babcock, E. B., and Collins, J. L. (1929). Does natural ionizing radiation control rate of mutation? Proc. Natl. Acad. Sci. U.S.A. 15, 623-628. doi: 10.1073/pnas.15.8.623

Baskerville, C. (1905). "Radium and radio-active substances. Their application especially to medicine," in The Physiological Action of Radio-Active Substances and their Therapeutic Applications (Philadelphia: Williams, Brown \& Earle).

Buesseler, K., Aoyama, M., and Fukasawa, M. (2011). Impacts of the Fukushima nuclear power plants on marine radioactivity. Environ. Sci. Technol. 45, 99319935. doi: 10.1021/es202816c

Chen, G. A., Gharib, T. G., Huang, C. C., Taylor, J. M., Misek, D. E., Kardia, S. L., et al. (2002). Discordant protein and mRNA expression in lung adenocarcinomas. Mol. Cell. Proteomics 1, 304-313. doi: 10.1074/mcp.M200008-MCP200

Danchenko, M., Skultety, L., Rashydov, N. M., Berezhna, V. V., Mátel, L., Salaj, T., et al. (2009). Proteomic analysis of mature soybean seeds from the Chernobyl area suggests plant adaptation to the contaminated environment. J. Proteome Res. 8, 2915-2922. doi: 10.1021/pr900034u

Dubinin, N. P., Vaulina, E. N., Kosikov, K. V., Anikeeva, I. D., Moskvitin, E. V., Zapadnaya, A. A., et al. (1973). Effects of space flight factors on the heredity of higher and lower plants. Life Sci. Space Res. 11, 105-110.

Gager, C. S. (1908). "Effects of the rays of radium on plants," in Memoirs of the New York Botanical Garden, Vol. 4 (Lancaster, PA: The New Era Printing Company), 1-278.

Gicquel, M., Esnault, M. A., Jorrin-Novo, J. V., and Cabello-Hurtado, F. (2011). Application of proteomics to the assessment of the response to ionising radiation in Arabidopsis thaliana. J. Proteomics 74, 1364-1377. doi: 10.1016/j.jprot.2011.03.025

Goodspeed, T. H., and Olson, A. R. (1928). The production of variation in Nicotiana species by X-ray treatment of sex cells. Proc. Natl. Acad. Sci. U.S.A. 14, 66-69. doi: 10.1073/pnas.14.1.66

Griffin, T. J., Gygi, S. P., Ideker, T., Rist, B., Eng, J., Hood, L., et al. (2002). Complementary profiling of gene expression at the transcriptome and proteome levels in Saccharomyces cerevisiae. Mol. Cell. Proteomics 1, 323-333. doi: 10.1074/mcp.M200001-MCP200

Guipaud, O., Holler, V., Buard, V., Tarlet, G., Royer, N., Vinh, J., et al. (2007). Time-course analysis of mouse serum proteome changes following exposure of the skin to ionizing radiation. Proteomics 7, 3992-4002. doi: 10.1002/pmic. 200601032

Hajduch, M., Casteel, J. E., Hurrelmeyer, K. E., Song, Z., Agrawal, G. K., and Thelen, J. J. (2006). Proteomic analysis of seed filling in Brassica napus. Developmental characterization of metabolic isozymes using high-resolution two-dimensional gel electrophoresis. Plant Physiol. 141, 32-46. doi: 10.1104/pp.105.075390

Hajduch, M., Ganapathy, A., Stein, J. W., and Thelen, J. J. (2005). A systematic proteomic study of seed filling in soybean. Establishment of high-resolution two-dimensional reference maps, expression profiles, and an interactive

\section{Supplementary Material}

The Supplementary Material for this article can be found online at: http://journal.frontiersin.org/article/10.3389/fpls.2015.00493

FIGURE S1 | The location of the Chernobyl experimental fields. The radio-contaminated field $(\mathrm{R})$ was established $5 \mathrm{~km}$ from Chernobyl Nuclear Power Plant $(C)$, within the exclusion zone (D). The non-radioactive field (C1) was initially established in 2007 about $100 \mathrm{~km}$ from C (A) but since 2008 the non-radioactive field (C2) has been transferred directly to the town of Chernobyl (B).

proteome database. Plant Physiol. 137, 1397-1419. doi: 10.1104/pp.104. 056614

Hajduch, M., Hearne, L. B., Miernyk, J. A., Casteel, J. E., Joshi, T., Agrawal, G. K., et al. (2010). Systems analysis of seed filling in Arabidopsis: using general linear modeling to assess concordance of transcript and protein expression. Plant Physiol. 152, 2078-2087. doi: 10.1104/pp.109.152413

Hayashi, G., Shibato, J., Imanaka, T., Cho, K., Kubo, A., Kikuchi, S., et al. (2014). Unraveling low-level gamma radiation-responsive changes in expression of early and late genes in leaves of rice seedlings at litate village, Fukushima. J. Heredity 105, 723-738. doi: 10.1093/jhered/esu025

Hornshoj, H., Bendixen, E., Conley, L. N., Andersen, P. K., Hedegaard, J., Panitz, F., et al. (2009). Transcriptomic and proteomic profiling of two porcine tissues using high-throughput technologies. BMC Genomics 10:30. doi: 10.1186/14712164-10-30

Houston, N. L., Hajduch, M., and Thelen, J. J. (2009). Quantitative proteomics of seed filling in castor: comparison with soybean and rapeseed reveals differences between photosynthetic and non-photosynthetic seed metabolism. Plant Physiol. 151, 857-868. doi: 10.1104/pp.109.141622

Hurlbert, S. H. (1984). Pseudoreplication and the design of ecological field experiments. Ecol. Monogr. 54, 187-211. doi: 10.2307/1942661

Karam, P. A., and Leslie, S. A. (2005). "Changes in terrestrial natural radiation levels over the history of life," in Natural Radiation Environment, Vol. 7, Proceedings of an International Symposium, Rhodes, Greece, 20-24 May 2002, eds J. P. McLaughlin, S. E. Simopoulos, and F. Steinhäusler (Dordrecht: Elsevier), 107-117.

Kinoshita, N., Sueki, K., Sasa, K., Kitagawaa, J, Ikarashia, S., Nishimura, T., et al. (2011). Assessment of individual radionuclide distributions from the Fukushima nuclear accident covering central-east Japan. Proc. Natl. Acad. Sci. U.S.A. 108, 19526-19529. doi: 10.1073/pnas.1111724108

Klubicova, K., Danchenko, M., Skultety, L., Berezhna, V. V., Rashydov, N. M., and Hajduch, M. (2013). Radioactive chernobyl environment has produced highoil flax seeds that show proteome alterations related to carbon metabolism during seed development. J. Proteome Res. 12, 4799-4806. doi: 10.1021/pr $400528 \mathrm{~m}$

Klubicova, K., Danchenko, M., Skultety, L., Berezhna, V. V., Uvackova, L., Rashydov, N. M., et al. (2012a). Soybeans grown in the chernobyl area produce fertile seeds that have increased heavy metal resistance and modified carbon metabolism. PLoS ONE 7:e48169. doi: 10.1371/journal.pone.0048169

Klubicova, K., Vesel, M., Rashydov, N. M., and Hajduch, M. (2012b). Seeds in Chernobyl: the database on proteome response on radioactive environment. Front. Plant Sci. 3:231. doi: 10.3389/fpls.2012.002313

Klubicova, K., Danchenko, M., Skultety, L., Miernyk, J. A., Rashydov, N. M., Berezhna, V. V., et al. (2010). Proteomics analysis of flax grown in Chernobyl area suggests limited effect of contaminated environment on seed proteome. Environ. Sci. Technol. 44, 6940-6946. doi: 10.1021/ es100895s

Kovalchuk, I., Abramov, V., Pogribny, I., and Kovalchuk, O. (2004). Molecular aspects of plant adaptation to life in the Chernobyl zone. Plant Physiol. 135, 357-363. doi: 10.1104/pp.104.040477

Kovalchuk, O., Burke, P., Arkhipov, A., Kuchma, N., James, S. J., Kovalchuk, I., et al. (2003). Genome hypermethylation in Pinus silvestris of Chernobyl a mechanism for radiation adaptation? Mutat. Res. 529, 13-20. doi: 10.1016/S0027-5107(03)00103-9 
Leszczynski, D. (2014). Radiation proteomics: a brief overview. Proteomics 14, 481-488. doi: 10.1002/pmic.201300390

Mimura, T., Mimura, M., Kobayashi, D., Komiyama, C., Sekimoto, H., Miyamoto, M., et al. (2014). Radioactive pollution and accumulation of radionuclides in wild plants in Fukushima. J. Plant Res. 127, 5-10. doi: 10.1007/s10265-013-0599-6

Moller, A. P., and Mousseau, T. A. (2013). The effects of natural variation in background radioactivity on humans, animals and other organisms. Biol. Rev. 88, 226-254. doi: 10.1111/j.1469-185X.2012.00249.x

Moller, A. P., and Mousseau, T. A. (2015). Strong effects of ionizing radiation from Chernobyl on mutation rates. Sci. Rep. 5, 8363. doi: 10.1038/srep08363

Monobe, M., Uzawa, A., Hino, M., Ando, K., and Kojima, S. (2005). Glycine betaine, a beer component, protects radiation-induced injury. J. Radiat. Res. 46, 117-121. doi: 10.1269/jrr.46.117

Nadson, G. A., and Philippov, G. S. (1925). Influence des rayons x sur la sexualité et la formation des mutantes chez les champignons inferieurs. C. R. Soc. Biol. Paris 93, 473-474.

Olson, A. R., and Gilbert, L. N. (1928). Natural reactivity and the origin of species. Nature 121, 673-674. doi: 10.1038/121673a0

Orntoft, T. F., Thykjaer, T., Waldman, F. M., Wolf, H., and Celis, J. E. (2002). Genome-wide study of gene copy numbers, transcripts, and protein levels in pairs of non-invasive and invasive human transitional cell carcinomas. Mol. Cell. Proteomics 1, 37-45. doi: 10.1074/mcp.M100019-MCP200

Park, E. C., Yoon, J. B., Seong, J. S., Choi, K. S., Kong, E. S., Kim, Y. J., et al. (2006). Effect of ionizing radiation on rat tissue: proteomic and biochemical analysis. Prep. Biochem. Biotechnol. 36, 19-35. doi: 10.1080/10826060500388470

Parnell, J. (2004). Mineral radioactivity in sands as a mechanism for fixation of organic carbon on the early Earth. Orig. Life Evol. Biosph. 34, 533-547. doi: 10.1023/B:ORIG.0000043132.23966.a1

Pascal, L. E., True, L. D., Campbell, D. S., Deutsch, E. W., Risk, M., Coleman, I. M., et al. (2008). Correlation of mRNA and protein levels: cell type-specific gene expression of cluster designation antigens in the prostate. BMC Genomics 9:246. doi: 10.1186/1471-2164-9-246

Rajjou, L., Belghazi, M., Huguet, R., Robin, C., Moreau, A., Job, C., et al. (2006). Proteomic investigation of the effect of salicylic acid on Arabidopsis seed germination and establishment of early defense mechanisms. Plant Physiol. 141, 910-923. doi: 10.1104/pp.106.082057

Rakwal, R., Agrawal, G. K., Shibato, J., Imanaka, T., Fukutani, S., Tamogami, S., et al. (2009). Ultra low-dose radiation: stress responses and impacts using rice as a Grass Model. Int. J. Mol. Sci. 10, 1215-1225. doi: 10.3390/ijms100 31215

Rashydov, N. M., and Malinovskiy, Y. Y. (2002). Migration of radionuclides from the "hot" particles to the root behavior zone in the soil and their accumulation in the cultural plants. Physiol. Biochem. Cult. Plants 34, 132-137.

Regente, M., and De La Canal, L. (2001). Are storage 2 S albumins also defensive proteins? Physiol. Mol. Plant Pathol. 59, 275-276. doi: 10.1006/pmpp.2001. 0365

Richards, A. (1915). Recent studies on the biological effects of radioactivity. Science (New York, N.Y.) 42, 287-300. doi: 10.1126/science.42.1079.287

Sahr, T., Voigt, G., Schimmack, W., Paretzke, H. G., and Ernst, D. (2005). Lowlevel radiocaesium exposure alters gene expression in roots of Arabidopsis. New Phytol. 168, 141-148. doi: 10.1111/j.1469-8137.2005.01485.x

Sales, M. P., Gerhardt, I. R., Grossi-De-Sa, M. F., and Xavier, J. (2000). Do legume storage proteins play a role in defending seeds against bruchids? Plant Physiol. 124, 515-522. doi: 10.1104/pp.124.2.515

Shkvarnikov, P. K. (1990). A cytological study of plants growing under exposure to different radiation levels. Tsitol. Genet. 24, 33-37.

Waditee, R., Bhuiyan, N. H., Hirata, E., Hibino, T., Tanaka, Y., Shikata, M., et al. (2007). Metabolic engineering for betaine accumulation in microbes and plants. J. Biol. Chem. 282, 34185-34193. doi: 10.1074/jbc.M704939200

Wellman, C. H., and Gray, J. (2000). The microfossil record of early land plants. Philos. Trans. R. Soc. Lond. B Biol. Sci. 355, 717-731. doi: 10.1098/rstb.2000. 0612

Yasunari, T. J., Stohl, A., Hayano, R. S., Burkhart, J. F., Eckhardt, S., and Yasunari, T. (2011). Cesium-137 deposition and contamination of Japanese soils due to the Fukushima nuclear accident. Proc. Natl. Acad. Sci. U.S.A. 108, 19530-19534. doi: $10.1073 /$ pnas. 1112058108

Conflict of Interest Statement: The authors declare that the research was conducted in the absence of any commercial or financial relationships that could be construed as a potential conflict of interest.

Copyright $\odot 2015$ Rashydov and Hajduch. This is an open-access article distributed under the terms of the Creative Commons Attribution License (CC BY). The use, distribution or reproduction in other forums is permitted, provided the original author(s) or licensor are credited and that the original publication in this journal is cited, in accordance with accepted academic practice. No use, distribution or reproduction is permitted which does not comply with these terms. 